\title{
A Novel Method of Constructing an Electroosmotic Micromixer with Double-Side Electrodes for Biotechnological Applications
}

\author{
Nima Talebzadeh ${ }^{*}$, Farshid Taleb Sis ${ }^{1}$, Siavash Zargari² and Hadi Veladi ${ }^{2}$ \\ Microsystem Fabrication Lab, Faculty of Electrical and Computer Engineering, \\ University of Tabriz, 29 Bahman Blvd, Tabriz, Iran \\ 'BUMEMS Lab, Department of Electrical and Electronic Engineering, Bogazici University, \\ Istanbul, Turkey \\ ${ }^{2}$ Faculty of Electrical and Computer Engineering, University of Tabriz, \\ 29 Bahman Blvd, Tabriz, Iran
}

(Received January 6, 2015; accepted April 24, 2015)

Key words: microfluidics, electroosmotic micromixer, double-side and single-side electrode configurations, mixing efficiency, misalignment

In this study, we provide a simple, low-cost, and novel method of constructing microelectrodes on both sides of a fluid microchannel to be utilized as an electroosmotic micromixer. The electrodes mounted on both sides of the microchannel provide unique characteristics for biological studies, electrokinetics, dielectrophoresis, and so forth. The fabrication process includes optical lithography, layer deposition to create electrodes, accurate alignment, and bonding of two wafers. Compared with other methods used for fabricating electrodes on both sides of microchannels, the method proposed in this study does not require very difficult and complex experimental conditions, cumbersome processes and expensive equipment. The distribution of the electric field is numerically analyzed for two electrode arrangements. The numerical analysis shows that the efficiency of the electrodes mounted on both sides of the microchannel is higher than that of the electrodes mounted on one side. The proposed technique is a good alternative to the one-side electrodes and other double-side electrode configurations for electroosmotic micromixers. The process simplicity provides an easy way of constructing microelectrodes on both sides of the microchannel for mixing fluids.

\section{Introduction}

As a result of progressive achievements in novel fabrication methods, fluid manipulation technologies and microfludic devices, such as lab-on-a-chip, and micrototal

${ }^{*}$ Corresponding author: e-mail: nima.talebzade@gmail.com 
analyses systems ( $\mu$ TAS) are at the forefront of the most dynamic segment of microelectromechanical systems (MEMS) technology. According to a study at MIT as early as 2004, microfluidics was named as one of the 10 emerging technologies that will transform our world.(1) Indeed, based on the Web of Science and USPTO databases, the number of publications involving microfluidics has markedly increased in the last decade.(2)

With embedded flexible membranes and hydraulic control microchannels, the degree of integration that can be achieved with this technology is impressive, and the total number of valves as switches that can be built per unit surface has grown exponentially. The growth rate of microfluidic valve density using microfluidic large-scale integration (mLSI) technology is four times faster than Moore's law predictions for integrated circuits. $^{(3,4)}$ With increasing interest of the microfluidics community in developing complete lab-on-a-chip solutions based on thin and flexible films, the principles of labon-a-chip are combined with foil technologies to obtain so-called lab-on-a-foil systems. ${ }^{(5)}$ Thin and flexible films as functional base materials enable various features.

Efficient and rapid mixing of fluids is an important and fundamental task in microfluidic applications such as blood typing, medical and chemical analyses, and biological research. ${ }^{(6)}$ Owing to the small size of microfluidic devices, fluid flow behaves as a low Reynolds number regime, which affects the mixing task when the fluid shows laminar behavior. This means that fluids refuse to mix efficiently. Micromixers are therefore employed to enhance the mixing rate at different flow rates. Indeed, micromixers as gradient generators have a number of novel biological applications in assays based on concentration gradients, such as studies of cancer metastasis and stem cell growth and differentiation. ${ }^{(7,8)}$ They are also defined as a labeling unit in immunoassays and immunomagnetic cell sorting (IMCS) systems. ${ }^{(9-11)}$ Broadly speaking, these devices are categorized as either passive or active, depending on the mixing strategy that they employ. Specific channel geometry configurations are used in passive micromixers to increase the surface contact area between the mixing species. In active devices, an external driving force or external mechanical components are employed to agitate the solution. Passive micromixers are further categorized as having parallel lamination, sequential lamination, sequential segmentation, segmentation based on injection, or focusing using a wide variety of microchannel structures. ${ }^{(12-21)}$ Active micromixers are classified as pneumatic, magnetic, acoustic, electrothermal, optical or electrokinetic. ${ }^{(12,22-24)}$

Electrokinetics generally involves the study of liquids or particle motion under the action of an electric field. Electroosmotic flow is an efficient method for mixing in microfluidic devices. No moving part is required in electroosmotic micromixers to disturb the fluid flow, and this is its distinct advantage. According to a study of some commercialized microfluidic point-of-care diagnostic devices, the electrokinetic micromixer is the most popular micromixer among the active ones. ${ }^{(10)}$ In this type of micromixer, the force applied to the fluid flow is based on the electric double layer (EDL) phenomenon. Owing to the ionization of surface groups and the electrolyte ion adsorption to solid surfaces, a thin layer with net charges is formed near the solid-liquid interface. This thin layer is called EDL. The EDL generally consists of two layers: one is the inner compact layer, where ions are strongly attracted to the charged surface, and 
the other is the diffuse layer, where ions are free to move and may be affected by an external electric field. These two layers are separated by a shear plane. The potential at this shear plane is known as the zeta potential ( $)$, and the thickness of the EDL is known as the Debye length $(\lambda)$. Aqueous solutions can be effectively driven by applying an electric force to the charges accumulated in the EDL causing fluid motion.

Sasaki et al.(25) describe the development of an electroosmotic micromixer. In this study, on the bottom surface of a channel, a pair of electrodes having a sinusoidal inter electrode gap was arranged in parallel. The AC voltage applied to the electrodes was increased to $20 V_{\mathrm{p}-\mathrm{p}}$, and frequency was varied from 1 to $5 \mathrm{kHz}$. Huang et al. (26) show a novel design of an electroosmotic micromixer with various electrode configurations on the bottom surface of a mixing chamber, which generated microvortex patterns with counter clockwise and clockwise rotations for the fluids in steady state. Park et al. ${ }^{(27)}$ describe the dependence of mixing performance on the number of electrode pairs. In this study, the micromixer utilizes asymmetric vortex flows generated by dcbiased ac electroosmosis (ACEO) for mixing. Park and Song illustrated that there may be an appropriate number of electrode pairs for a given flow rate for the best mixing performance, because both flow rate and number of electrode pairs affect the development of vortices and, subsequently, the mixing performance. Using an electrode configuration on both side walls or bottom and top surfaces of a mixing channel is another strategy in electroosmotic micromixers, which has been rarely studied, and investigations on electroosmotic micromixers with double-side electrodes are mostly only based on numerical simulations and analyses. In ref. 28, numerical simulations of an electrokinetic micromixer are presented. Four electrodes on the upper and lower surfaces of the mixing chamber were mounted to apply a chaotic electric field to the fluid flow. Similar numerical simulations for this type of electrokinetic micromixers have also been reported. ${ }^{(29-31)}$

The ability to construct microelectrodes on both sides of a lab-on-a-chip channel has led to a variety of very new areas in microfluidic technology. The electrode mounted on one side of the microchannel lacks the advantages of electrodes mounted on both sides. The high electric field gradient produced in this type of electrode arrangement causes a stronger electrokinetic force, leading to faster response, improved performance and high miniaturization. The most important features and applications of electrode arrangements on both sides of the microchannel are more accurate estimation and detection of fluid volume, the presence of microparticles and biological cells, and their specifications using local impedance or capacitance measurements. ${ }^{(32)}$ There are numerous studies of electrode arrangements on more than one side of the microchannel, such as in the case of enzyme-linked immunosorbent assay (ELISA) and electrowetting on dielectrics (EWOD) technologies. ${ }^{(33,34)}$

In this study, we provide a new, low-cost, and simple biocompatible method of constructing an electroosmotic micromixer with electrodes on both sides of the microchannel. To illustrate the importance of this type of micromixer, an electroosmotic micromixer with electrodes on both sides of the channel (top and bottom) is compared with a micromixer with electrodes fabricated on one side of the channel (bottom) with the help of COMSOL Multiphysics. Then, a soft-lithography technique is used to create 
an electroosmotic micromixer with electrodes on both sides of the microchannel. Finally, the effect of misalignment caused by a SU-8 layer is investigated. The main advantages of the proposed process include cost-effectiveness, ease of construction, fast response, high efficiency of the micromixer, and small dimensions of the chip.

\section{Materials and Methods}

\subsection{Theoretical concepts}

The motion of fluids is governed by the Navier-Stokes equation, as shown below. Here, the equation contains a source term, which imposes the electroosmotic force on the flow: ${ }^{(35)}$

$$
\begin{gathered}
\nabla \cdot \boldsymbol{V}=0, \\
\rho \frac{\partial \boldsymbol{V}}{\partial t}+\rho \boldsymbol{V} \cdot \nabla \boldsymbol{V}=-\nabla \boldsymbol{P}+\mu \nabla^{2} \boldsymbol{V}+\rho_{\mathrm{e}} \boldsymbol{E},
\end{gathered}
$$

where $t$ is time, and $\boldsymbol{V}$ and $\boldsymbol{P}$ represent the fluid velocity and its pressure, respectively. $\mu$ is the fluid dynamic viscosity, $\rho$ is the density of charged particles within the flow, and $\boldsymbol{E}$ is the applied electric field. This gives rise to a finite volumetric net charge density $\left(\rho_{\mathrm{e}}\right)$ in the fluid close to the charged walls. Here, the charge density within the flow is obtained from the following equation:

$$
\rho_{\mathrm{e}}=-2 z e c_{0} \sinh \left(\frac{z e \psi}{k_{\mathrm{b}} T}\right)
$$

where $z$ is the valence of the ion, which is the same for the ions in a symmetric electrolyte solution, $e$ is the electrical charge of an electron, $c_{0}$ is the initial concentration of the bulk solution, $k_{\mathrm{b}}$ is the Boltzmann constant, $T$ is the absolute temperature, and $\psi$ indicates the potential distribution of the EDL. The force exerted on the fluid by the electric field is equal to $\rho_{\mathrm{e}} \boldsymbol{E}$. In other words,

$$
\boldsymbol{F}_{\mathrm{EOF}}=\rho_{\mathrm{e}} \boldsymbol{E} .
$$

The electrokinetic driving body force is defined as ${ }^{(28)}$

$$
\boldsymbol{F}_{\mathrm{EOF}}=\frac{-2 z e c_{0}}{\varepsilon} \sinh \left(\frac{z e \psi}{k_{\mathrm{b}} T}\right) \nabla(\boldsymbol{\psi}+\boldsymbol{\phi})
$$

where $\phi$ is the applied electrical potential. For a better understanding of the equations governing the behavior of microfluidics by electronics engineers, ref. 2 provides a table of the dual equivalent symbols used in electronics and fluid mechanics. When we are dealing with microfluids, the law of conservation of mass is equivalent to the charge conservation law in electronics. Ohm's law also holds in fluid mechanics. 


\subsection{Structure of the proposed micromixer}

Figures 1(a)-1(c) show the three-dimensional configuration and dimensions of the proposed micromixer, the two-dimensional cross-sectional area, and the top view, respectively. The inlet width of each fluid is $50 \mu \mathrm{m}$ entering a $100-\mu \mathrm{m}$-thick channel. Two pairs of electrodes are mounted on both sides of the channel (on the top and bottom) to apply an electric field for mixing the fluids. The width of each electrode is $50 \mu \mathrm{m}$ installed at a constant distance of $50 \mu \mathrm{m}$. The red and blue electrodes show the positive and negative voltage polarities, respectively. The top and bottom substrates are Pyrex of $3 \mathrm{~mm}$ thickness. The alignment marks were designed on one of the substrates as a "plus" mark with a branch width of $100 \mu \mathrm{m}$ and a length of $1.5 \mathrm{~mm}$. The complementary alignment marks were designed on another substrate with a width of $1.5 \mathrm{~mm}$ to align the two wafers.

The fluid in the micromixer is two aqueous solutions. Its mechanical and electrical properties and the initial values of parameters are presented in Table 1. ${ }^{(36)}$

\section{Experimental}

(A) First, a copper seed layer is deposited on the Pyrex wafer by sputtering. For this purpose, a 40-nm-thick $\mathrm{Cr}$ layer as the adhesive layer is first deposited to adhere the copper and Pyrex layers. Then, a 100-nm-thick copper layer is deposited. The photoresist is then deposited on the wafer and is patterned using a suitable mask, UV radiation, and a specific developer. The wafer is then placed into a metal solution

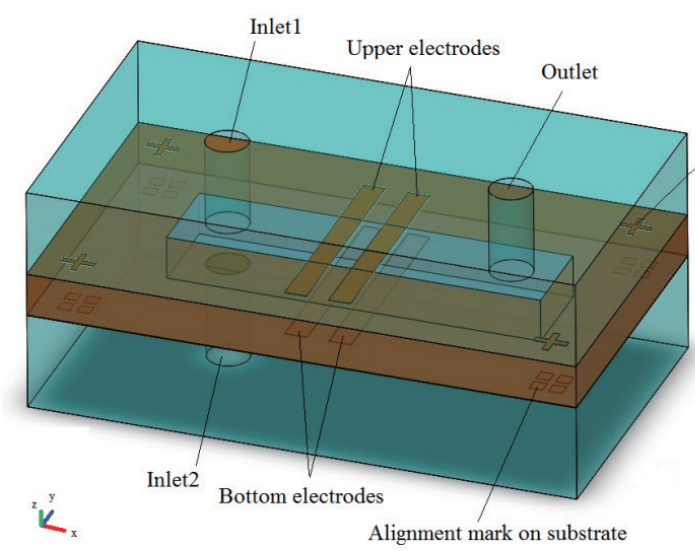

(a)

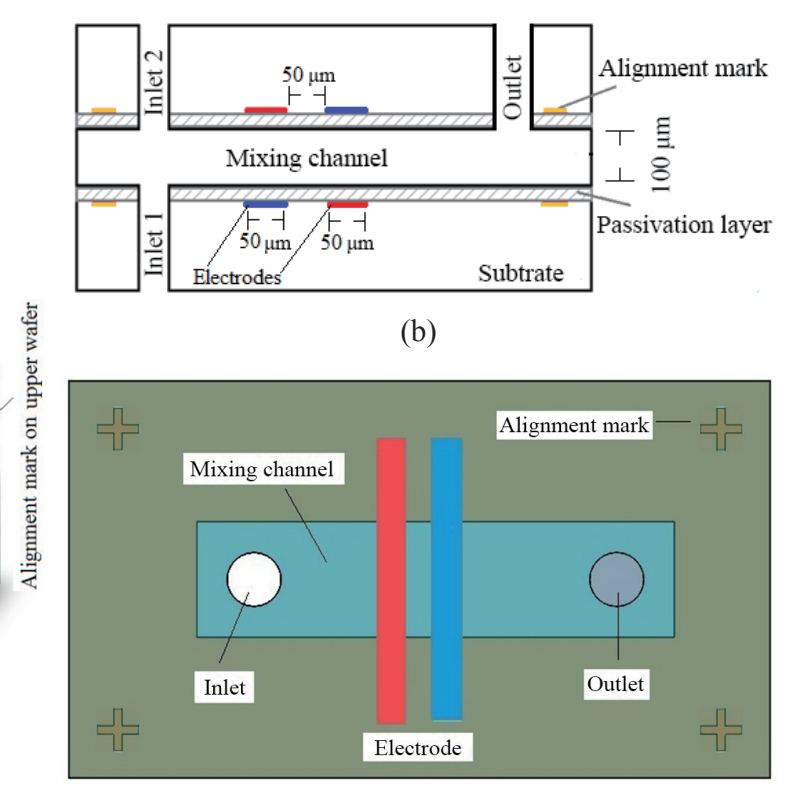

(c)

Fig. 1. (Color online) Configuration and dimensions of the proposed micromixer, (a) threedimensional view, (b) cross-sectional area, and (c) top view. 
Table 1

Mechanical and electrical properties of the fluid and initial values of parameters. ${ }^{(36)}$

\begin{tabular}{lcc}
\hline Symbol & Value & Description \\
\hline$\rho$ & $1000\left(\mathrm{~kg} / \mathrm{m}^{3}\right)$ & Density \\
$\eta$ & $10^{-3}(\mathrm{~Pa} \cdot \mathrm{s})$ & Dynamic viscosity \\
$\varepsilon_{\mathrm{r}}$ & 80.2 & Relative permittivity of the fluid \\
$\sigma$ & $0.118(\mathrm{~S} / \mathrm{m})$ & Conductivity of the ionic solution \\
$\zeta$ & $-0.1(\mathrm{~V})$ & Zeta potential \\
$U_{0}$ & $0.1(\mathrm{~mm} / \mathrm{s})$ & Mean inflow velocity \\
$D$ & $10^{-11}\left(\mathrm{~m}^{2} / \mathrm{s}\right)$ & Diffusion coefficient of the solution \\
$C_{0}$ & $1\left(\mathrm{~mol} / \mathrm{m}^{3}\right)$ & Initial concentration of the fluid \\
$f$ & $20 \mathrm{~Hz}$ & Frequency applied to the electrodes \\
$V_{0}$ & $2 \mathrm{~V}$ & Amplitude of voltage applied to the electrodes \\
\hline
\end{tabular}

[iron(III) chloride etchant] so that only the desired parts of the seed layer remain, i.e., the electrodes and alignment marks. The wafer is placed in acetone to remove the photoresist material from the surface. Figure 2(a) shows the design at this stage.

(B) A very thin layer of polydimethysiloxane (PDMS) is deposited on the entire wafer to act as a protective layer to prevent the direct contact of the electrodes and fluid. Metals are not biocompatible materials and may change the nature of the biological cells in contact with them. Therefore, an insulating layer is usually placed to prevent the direct contact between the electrodes and the biological cells in biological applications. For this purpose, a PDMS layer is spun on the wafer at the desired speed to reach a thickness of $5 \mu \mathrm{m}$. The wafer is then allowed to cure in an oven for $20 \mathrm{~min}$. Figure 2(b) shows the design at this stage.

(C) SU-8 is a biocompatible polymeric material with many applications in microfluidic technology. Accordingly, SU-8 is used to fabricate the mixing channel. First, SU-8 is poured on the wafer and then spun at a proper speed according to the desired thickness of the mixing channel $(100 \mu \mathrm{m}$ in this study). Then, the pattern of the mixing channel is created on SU-8 using the appropriate mask, UV radiation, and the specific developer. Figure 2(c) shows the design at this stage.

(D) A new wafer is selected and stages (A) and (B) are repeated to fabricate a wafer with complementary alignment marks. Using several different techniques including lasers, the inlets and outlet are separately fabricated on both wafers. Then, the two wafers are bonded. To completely bond the two wafer surfaces, both wafers are irradiated with oxygen plasma. Then, the alignment marks are carefully aligned using an optical microscope. Thereafter, the wafers are bonded. The final structure of the micromixer is shown in Fig. 1(a).

One of the crucial steps in the proposed process is the exact alignment of the upper and lower wafers. This was carried out with the help of alignment marks on the wafers using an optical microscope with very high resolution and magnification. Misalignment will affect the performance of the micromixer. 


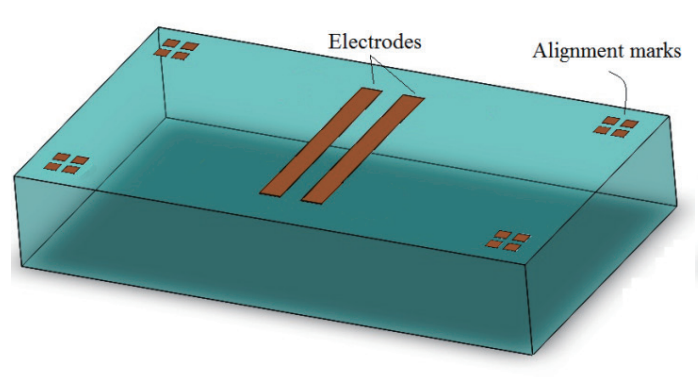

(a)

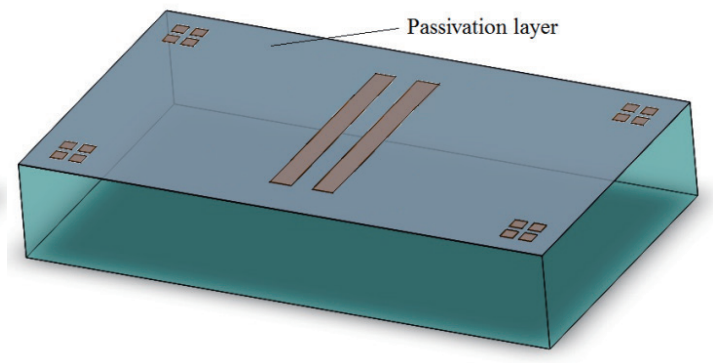

(b)

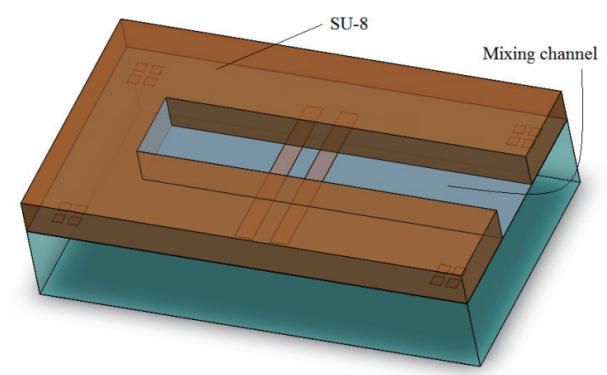

(c)

Fig. 2. (Color online) Fabrication steps of the proposed electroosmotic micromixer: (a) depositing and patterning the seed layer, (b) coating the bio-compatible layer, and (c) developing the mixing channel using SU-8.

\section{Results}

\subsection{Comparison of two electrode arrangements}

First, the electroosmotic micromixer is compared with the electrode arrangement on one side of the microchannel to analyze and demonstrate the advantages of the electroosmotic micromixer and the distribution of the electric field generated inside the microchannel equipped with double-side electrodes. Since most microfluidic devices are based on surface electrodes on only one side (usually the bottom of the channel), the electrokinetic force is mostly generated only around the electrodes. Moving from the electrode surface to the top of the channel, electrokinetic force decreases exponentially. Accordingly, more energy is required to affect the fluid or microparticles on the upper portion of the channel. Placing microelectrodes on the lower and upper portions of the channel largely resolves this problem. Thus, a large electrokinetic force is generated in the whole cross-sectional area of the channel. This will result in faster response, higher efficiency, and miniaturization of the microfluidic devices.

As mentioned in $\S 2.1$, the force exerted on the fluid by the electric field is equal to $\rho_{\mathrm{e}} \boldsymbol{E}$; eq. (5) shows the electroosmotic body force in more detail. As shown in eq. (4), the force applied to the fluid has a direct relationship with the applied electric field. When the electrodes are embedded on one side of the microchannel, the electric field lines generating a larger force are not uniformly distributed in the channel cross section and have a greater impact on the fluid only near the surface of the electrodes. The electric 
field lines are extremely weakened with increasing distance from the electrode surface to the upper portion of the channel. In the case where the electrodes are installed on both sides of the channel, the electric field is maximum on the surfaces of electrodes and minimum in the middle of the channel. In this type of electrode arrangement, the fluid experiences a much larger force in a given cross section.

Figure 3 shows the distribution of the normalized electric field inside a microchannel from the bottom to the top with a pair of electrodes embedded on the bottom (a) and both sides (top and bottom) (b). In simulations, the amplitude of the voltage applied to the electrodes in both cases is the same, and the electric field distribution is ranging from maximum (white) to minimum (black). As can be seen, the distribution of the normalized electric field in case (b) is much larger, and thus a greater force is exerted on the fluid in a certain cross section of the channel.

The efficiency and response speed of the proposed micromixer were evaluated. COMSOL Multiphysics is used for simulations and analysis of results, which has great potential for the coupling of different types of environments. The mixing efficiency expressed as a percentage at the microchannel outlet is determined using the following equation:(28)

$$
\left(1-\frac{\int_{\text {lower surface }}^{\text {upper surface }}\left|C-C_{\infty}\right| d y}{\int_{\text {lower surface }}^{\text {upper surface }}\left|C_{0}-C_{\infty}\right| d y}\right) \times 100,
$$

where $C$ is the concentration profile of species across the channel, $C_{\infty}$ is the concentration in the perfectly mixed state, and $C_{0}$ is the initial unmixed concentration. In this study, the voltage applied to the electrodes is a sinusoidal signal with an amplitude of $2 \mathrm{~V}$ and a frequency of $20 \mathrm{~Hz}$. Figure 4 shows the simulated concentration distribution and the steady-state velocity field within the channel.

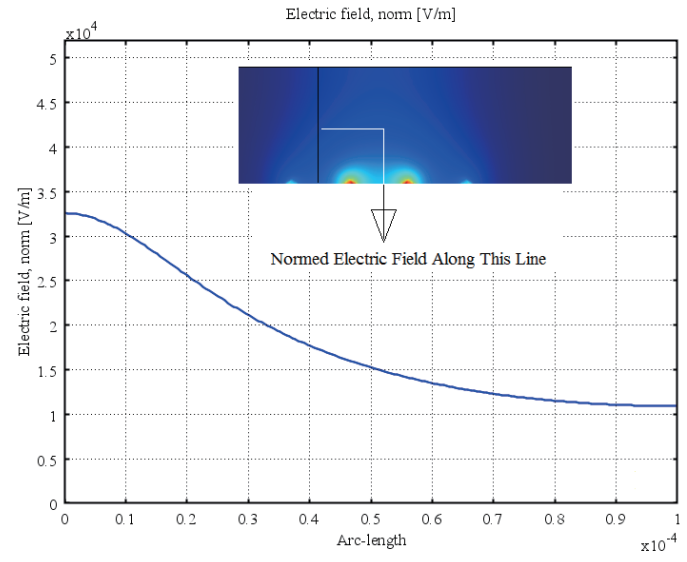

(a)

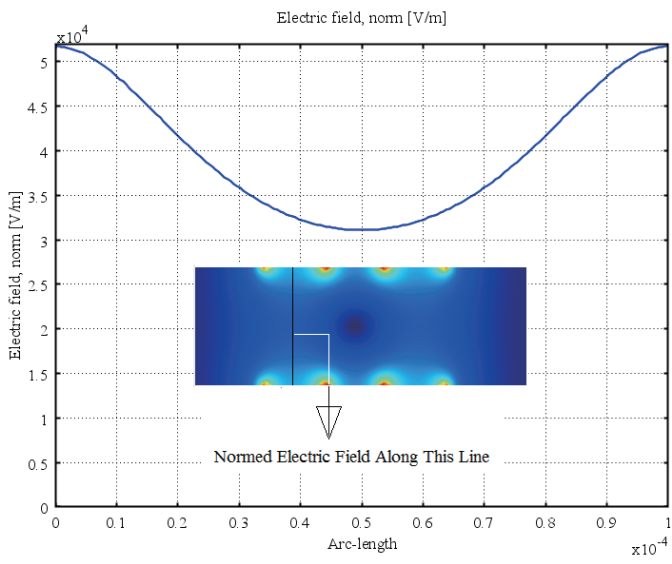

(b)

Fig. 3. (Color online) Distribution of the normalized electric field inside a microchannel from the bottom to the top (a) with a pair of electrodes on the bottom and (b) on both sides. 


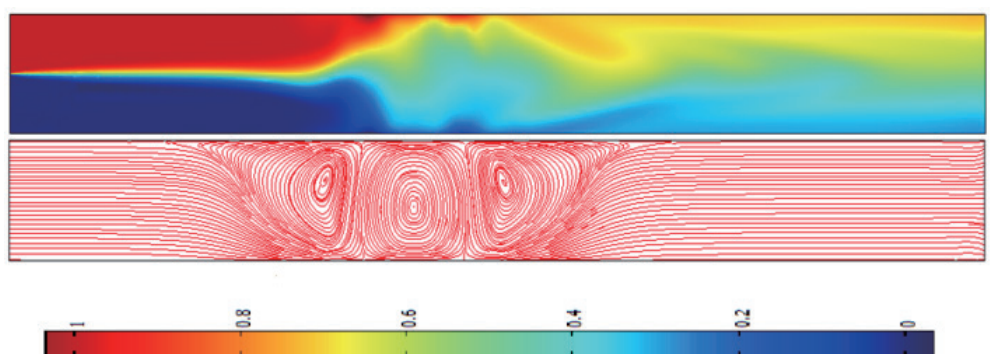

Fig. 4. (Color online) Concentration distribution and steady-state velocity field within the channel.

As can be seen, the eddy currents caused by the alternating voltage applied to the fluid result in turbulences and thus perfect mixing of two fluids. Figure 5 shows the transient response of the proposed micromixer in the range of 0 to $20 \mathrm{~s}$. As shown, the mixing efficiency of fluids is increased and approaches perfect mixing over time. Figure 6 shows the steady-state concentration profile at the outlet indicating the effectiveness of the micromixer. A steady-state efficiency of $75.6 \%$ is achieved for the micromixer using eq.(6).

\subsection{Impact of misalignment}

As described in the previous section, the exact alignment of two wafers using the alignment marks is the most important and most essential stage in the fabrication of the proposed micromixer. Note that due to a $100-\mu \mathrm{m}$-thick layer between the upper and lower wafers, there is a high potential for misalignment. With respect to the accuracy of the microscope, misalignment is inevitable. The misalignment between the two wafers may occur in the bonding step in both $x$ - and $y$-directions. Misalignment in the $y$-direction is not a problem, because the electrodes are opposite. Thus, the electric field between the pair of opposite electrodes is perpendicular to the fluid flow direction. When there is a misalignment in the $x$-direction, the electrodes are not completely opposite, and thus the electric field between the opposite electrodes is not perpendicular to the direction of fluid flow. This weakens the force applied to the fluid and thus reduces the efficiency of the micromixer. Figure 7 shows how misalignment occurs during the fabrication of the proposed electroosmotic micromixer.

For an intuitive understanding of the problem, Fig. 8 shows the voltage distribution inside the channel and the vector electric field direction in the middle of the channel for a misalignment of $80 \mu \mathrm{m}$. As can be seen, for a misalignment of $80 \mu \mathrm{m}$ between the two opposite electrodes, the electric field is not perpendicular to the flow direction, but is tilted, weakening the force exerted on the fluid.

Figure 9 shows the simulated concentration distribution for misalignment of the opposite electrodes from 0 to $100 \mu \mathrm{m}$ with an incremental step of $20 \mu \mathrm{m}$. Figure 10 shows the transient response for six electrode shifts from 0 to $20 \mathrm{~s}$. Figure 11 illustrates the steady-state response of the proposed micromixer for an electrode shift up to $100 \mu \mathrm{m}$ [the results were obtained using eq. (6)]. As expected, with increasing electrode shift, 


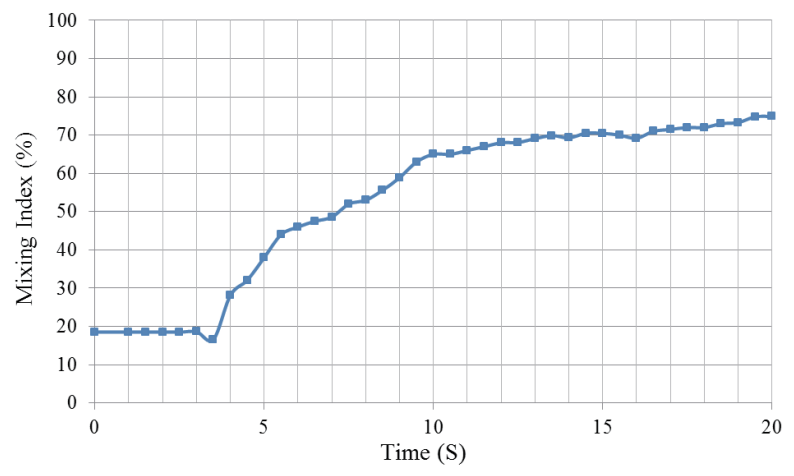

Fig. 5. (Color online) Transient response of the proposed micromixer in the range of 0 to $20 \mathrm{~s}$.

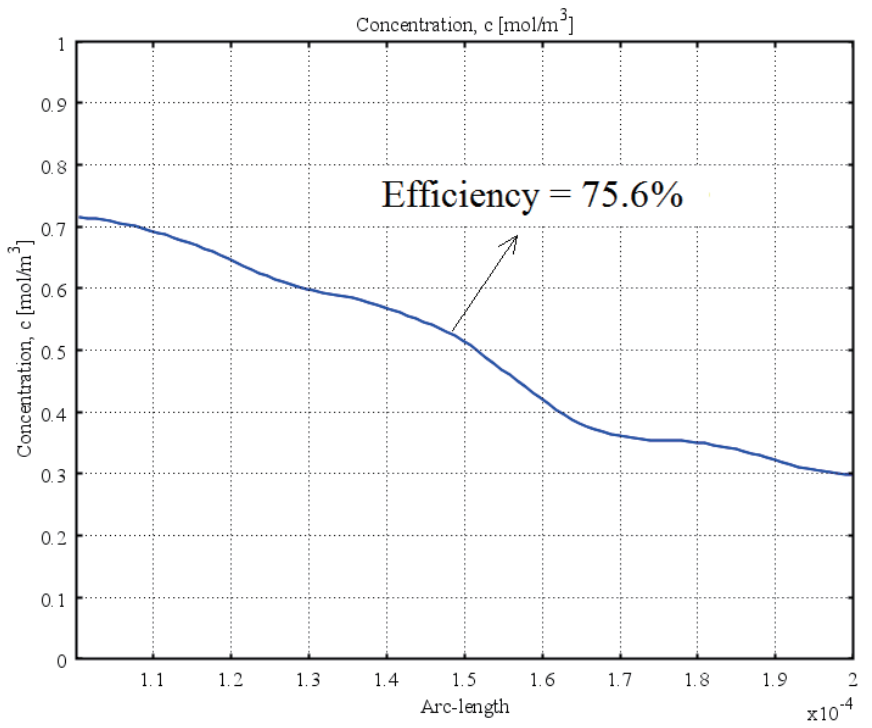

Fig. 6. (Color online) Steady-state concentration profile at the outlet of the micromixer.

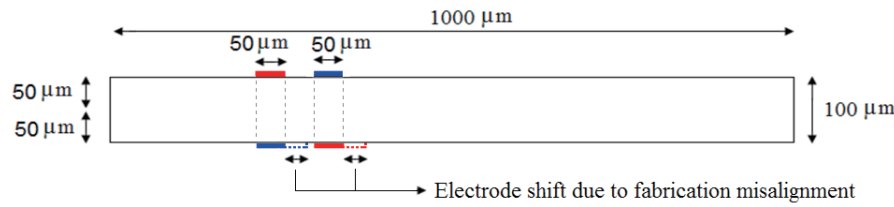

Fig. 7. (Color online) Concept of fabrication misalignment. 


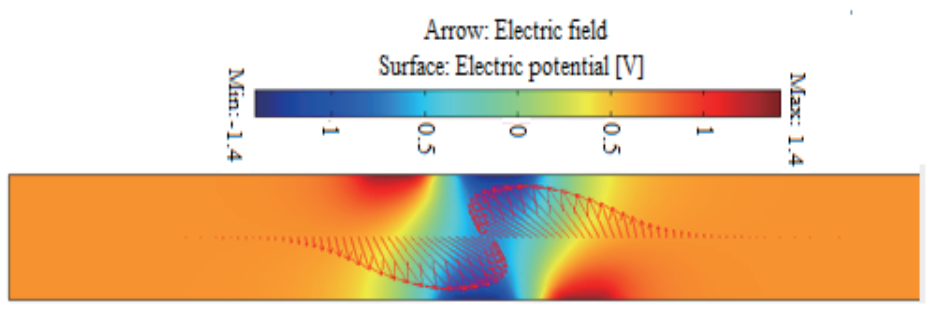

Fig. 8. (Color online) Distribution of voltage inside the channels as color distribution and direction of the electric field in the middle of the channel as shown by vectors for a misalignment of $80 \mu \mathrm{m}$.

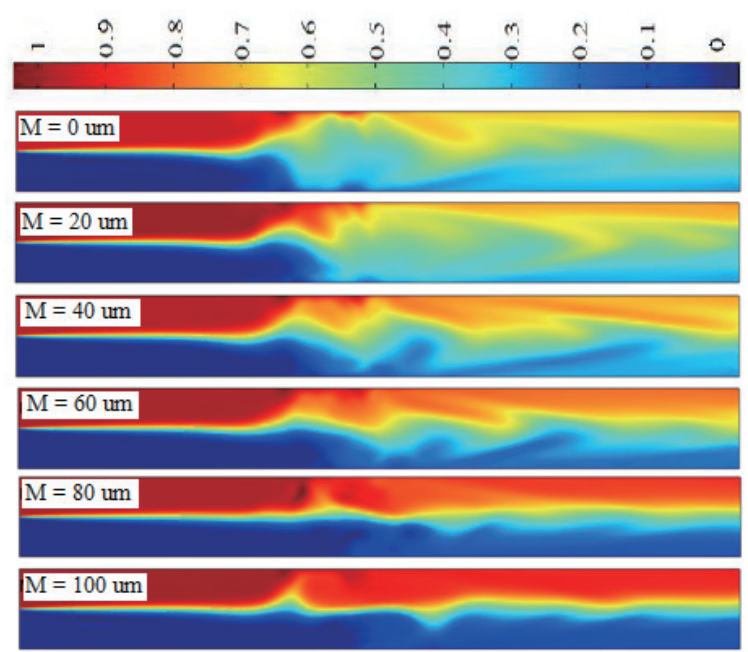

Fig. 9. (Color online) Concentration distribution for misalignment of opposite electrodes from 0 to $100 \mu \mathrm{m}$ with incremental steps of $20 \mu \mathrm{m}$.

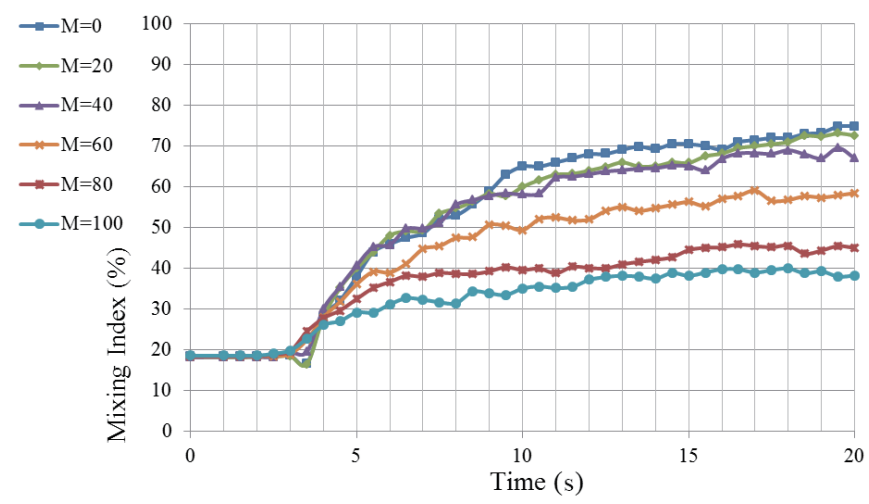

Fig. 10. (Color online) Transient response for six electrode shifts from 0 to $20 \mathrm{~s}$. 


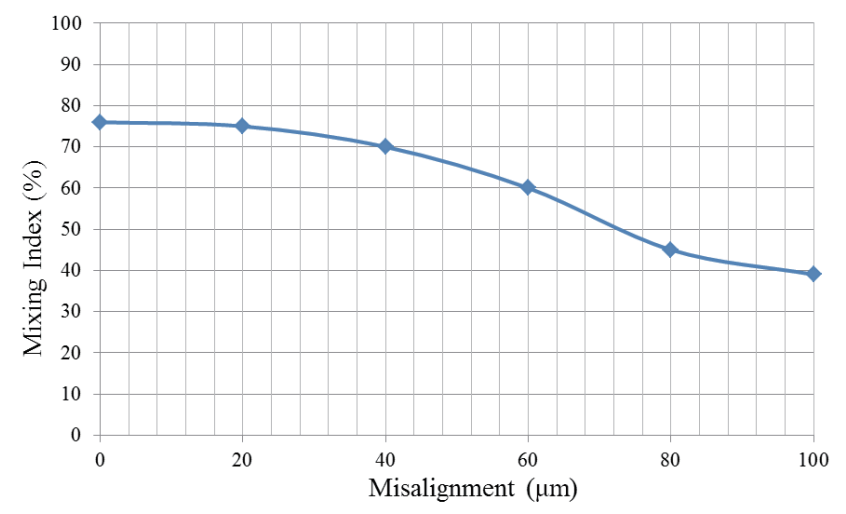

Fig. 11. (Color online) Steady-state response of the proposed micromixer for an electrode shift up to $100 \mu \mathrm{m}$.

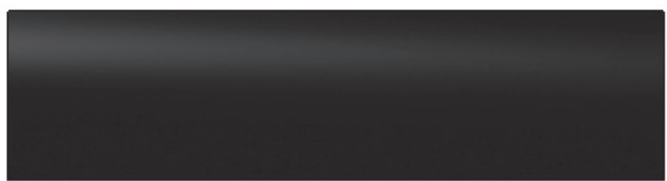

(a)

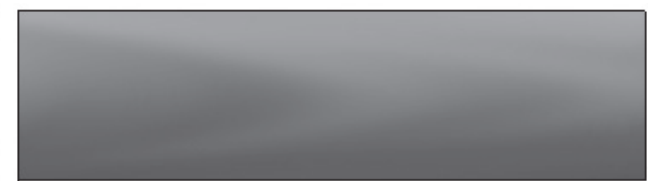

(b)

Fig. 12. Top view of the end portion of the mixing channel output before and after applying the electric field.

the electric field between the opposite electrodes deviates from the perpendicular mode, reducing the effective force on the fluid and thus reducing the mixing efficiency. As shown in Fig. 11, a small misalignment of the two wafers (up to $20 \mu \mathrm{m}$ ) has a reasonably low impact on the performance of the electroosmotic micromixer.

Figure 12 shows the top view of the end portion of the mixing channel representing the color distribution and mixing performance. In the image on the left, the electric field is not applied to the fluid, and the two fluids slip over each other without mixing. The white fluid flows at the bottom of the channel under the black fluid. Because the imaging is from the upper surface of the micromixer, only the black color (fluid A) can be seen. The two fluids are mixed after applying the electric field, as shown in the image on the right (one of the fluids is pure water and the other is black water).

\section{Conclusions}

In this paper, a novel, simple, cost-effective method with high integrability is proposed to fabricate an electroosmotic micromixer with electrodes on both sides of the mixing channel. The primary reason is that, against the single side electrode arrangement, the electric field of the double-side electrodes does not decay rapidly away from the substrate, resulting in much greater effective volume. The proposed process 
includes seed layer deposition, optical lithography, deposition of the protective layers on the electrodes, and exact alignment of the two wafers. Owing to the thick layer between the two wafers, misalignment during the fabricating process is inevitable. The impact of misalignment on the performance of the micromixer is negligible for misalignment less than $20 \mu \mathrm{m}$. The electroosmotic micromixer proposed in this study has potential biotechnological applications and can be a good alternative to a mixer with a one-side electrode arrangement. Moreover, the ease of fabrication and high integrability will lead to high time saving and cost-effectiveness of the electroosmotic micromixer.

\section{References}

1 G. Huang: MITS Technol. Rev. 107 (2004) 32.

2 D. E. Angelescu: Highly Integrated Microfluidics Design (Artech House, Boston, 2011).

3 J. W. Hong and S.R. Quake: Nat. Biotechnol. 21 (2003) 1179.

4 T. Thorsen, S. J. Maerkl and S. R. Quake: Science 298 (2002) 580.

5 M. Focke, D. Kosse, C. Müller, H. Reinecke, R. Zengerle and F. von Stetten: Lab Chip 10 (2010) 1365.

6 N. Nam-Trung: Micromixers Fundamentals, Design and Fabrication 2nd ed. (Elsevier, London, 2012) p. 321.

7 S. J. Wang, W. Saadi, F. Lin, C. Minh-Canh Nguyen and N. Li Jeon: Exp. Cell. Res. 300 (2004) 180.

8 B. G. Chung, L. A. Flanagan, S. W. Rhee, P. H. Schwartz, A. P. Lee, E. S. Monuki and N. L. Jeon: Lab Chip 5 (2005) 401.

9 C. Chaktranond, K. Fukagata and N. Kasagi: J. Fluid Sci. Technol. 3 (2008) 1008.

10 C. D. Chin, V. Linder and S. K. Sia: Lab Chip 12 (2012) 2118.

11 M. L. Sin, J. Gao, J. C. Liao and P. K. Wong: J. Biol. Eng. 5 (2011) 1.

12 N. Nam-Trung and S. T. Wereley: Fundamentals and applications of microfluidics (Artech House, Boston, 2006) p. 357.

13 T. Scherr, C. Quitadamo, P. Tesvich, D. Sang-Won Park, T. Tiersch, D. Hayes, J.-W. Choi, K. Nandakumar and W. T. Monroe: J. Micromech. Microeng. 22 (2012) 055019.

14 M. K. Parsa and F. Hormozi: J. Micromech. Microeng. 24 (2014) 065018.

15 D. Lin, F. He, Y. Liao, J. Lin, C. Liu, J. Song and Y. Cheng: J. Opt. 15 (2013) 025601.

16 C. K. Chung, C. C. Lai, T. R. Shih, E. C. Chang and S. W. Chen: Micro Nano Lett. 8 (2013) 567.

17 Y. Liu, Y. Deng, P. Zhang, Z. Liu and Y. Wu: J. Micromech. Microeng. 23 (2013) 075002.

18 Y. Murakami, K. Araki, R. Ohashi, H. Honma, N. Misawa, K. Takahashi, K. Sawada and M. Ishida: Sens. Actuators, B 194 (2014) 528.

19 H. Le The, H. Le-Thanh, N. Tran-Minh and F. Karlsen: Biomedical Engineering (MECBME) (IEEE, Middle East, 2014) pp. 25-28.

20 A. S. Yang, F. C. Chuang, C. L. Su, C. K. Chen and M. H. Lee: Mechatronics and Automation (ICMA) (IEEE, 2013) pp. 152-157.

21 W. S. Yoo, J.S. Go, S. Park and S. H. Park: J. Micromech. Microeng. 22 (2012) 035007.

22 H. Ukita: Micromechanical photonics (Springer, Shiga, 2007) p. 121.

23 M. Rahbar, L. Shannon and B. L. Gray: J. Micromech. Microeng. 24 (2014) 025003.

24 K. R. Huang, J.S. Chang, S. D. Chao, T. S. Wung and K. C. Wu: Jpn. J. Appl. Phys. 51 (2012) 047002.

25 N. Sasaki, T. Kitamori and H. B. Kim: Anal. Sci. 26 (2009) 815.

26 S. H. Huang, S. K. Wang, H. S. Khoo and F. G. Tseng: Sens. Actuators, B 125 (2007) 326. 
27 B. O. Park and S. Song: J. Micromech. Microeng. 22 (2012) 1.

28 C. C. Cho: Microfluid. Nanofluid. 5 (2008) 785.

29 M. Hadigol, R. Nosrati, A. Nourbakhsh and M. Raisee: J. Non-Newtonian Fluid Mech. 166 (2011) 965.

30 M. Jain, A. Yeung and K. Nandakumar: Micromachines 1 (2010) 36.

31 N. Talebzadeh and H. Veladi: Robotics and Mechatronics (IEEE, Tehran, 2014) p. 654.

32 M. Li, S. Li, W. Cao, W. Li, W. Wen and G. Alici: Microfluid. Nanofluid. 14 (2013) 527.

33 S. Numthuam, T. Kakegawa, T. Anada, A. Khademhosseini, H. Suzuki and J. Fukuda: Sens. Actuators, B 156 (2011) 637.

34 S. Morishita, M. Kubota and Y. Mita: Micro Electro Mechanical Systems (IEEE, 2012) p. 1045.

35 J. A. Pelesko and D. H. Bernstein: Modeling Mems and Nems (CRC press, London, 2002) p. 9.

36 A.Z. Kouzani, K. Khoshmanesh, S. Nahavandi and J. Kanwar: Systems, Man and Cybernetics (IEEE, 2009) p. 4618. 